\title{
Agenesis of the Bilateral Internal Carotid Arteries Led to the Development of Vertigo
}

\author{
Yasushi Shibata* \\ Department of Neurosurgery, Mito Medical Center, University of Tsukuba, Ibaraki, Japan
}

*Corresponding author: Yasushi Shibata, MD, PhD, Department of Neurosurgery, Mito Medical Center, University of Tsukuba, Ibaraki, Japan, E-mail: yshibata@md.tsukuba.ac.jp

\begin{abstract}
Agenesis of the bilateral internal carotid arteries is a rare disease. There have been few case reports of subjects who developed cerebral infarction, intracranial hemorrhage or subarachnoid hemorrhage. We experienced a rare case of agenesis of the bilateral internal carotid arteries in which the patient developed vertigo. A 63-year-old woman suddenly became dizzy one morning after waking up and was admitted to our hospital. Brain Computed Tomography (CT) and Magnetic Resonance (MR) imaging demonstrated no intracranial hemorrhage or cerebral infarction. CT angiography and MR angiography showed the enlargement and invagination of the basilar artery into the pons. Her dizziness disappeared with conservative therapy. Our case was very rare because the patient was diagnosed with agenesis of the bilateral internal carotid arteries before the development of major cerebrovascular disease. There are some possible mechanisms of vertigo in our case: One is direct pulsatile pontine compression by the elongated basilar artery; another is transient or chronic cerebral ischemia caused by abnormal circulation.
\end{abstract}

\section{Keywords}

Agenesis of bilateral internal carotid arteries, Vertigo

\section{Introduction}

Agenesis of the bilateral internal carotid arteries is a rare disease. There have been few case reports of subjects who developed cerebral infarction, intracranial hemorrhage or subarachnoid hemorrhage [1-4]. We experienced a rare case of agenesis of the bilateral internal carotid arteries in which the patient developed vertigo.

\section{Case Report}

The patient, a 63-year-old woman, had been trea- ted for hypertension and hyperlipidemia for 1 year. She developed breast pain and visited the Department of Surgery at our hospital. She was diagnosed with breast cancer, and breast surgery was scheduled. The patient suddenly became dizzy one morning after waking up and was admitted to our hospital. Her physical and neurological findings were almost normal. Brain Computed Tomography (CT, Figure 1) and Magnetic Resonance (MR) imaging (Figure 2) demonstrated no intracranial hemorrhage or cerebral infarction. CT angiography (Figure 3) and MR angiography (Figure 4) showed the enlargement and invagination of the basilar artery into the pons. The bilateral anterior and middle cerebral arteries were supplied from posterior circulation via the development of bilateral posterior communicating arteries. Skull CT showed the complete occlusion of the bilateral carotid canals (Figure 5). Cerebral angiography (Figure 6 and Figure 7) and carotid ultrasound confirmed agenesis of the bilateral internal carotid arteries. There was no collateral flow from extracranial arteries into the intracranial brain region. The bilateral ophthalmic arteries were supplied from extracranial arteries. No aneurysm or major arterial stenosis was found. Technetium-99m Ethyl Cysteinate Dimer (TCECD) Single Photon Emission CT (SPECT) showed mild low cerebral perfusion at the left parietal lobe (Figure 8). CT perfusion showed almost normal cerebral perfusion (Figure 9). Her dizziness disappeared with conservative therapy.

\section{Discussion}

There have been only several case reports of agenesis of the bilateral internal carotid arteries. Most of the-

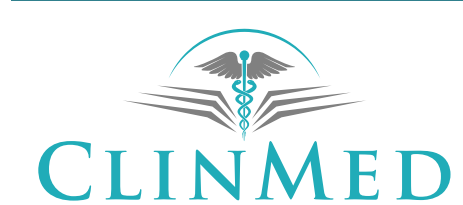

INTERNATIONAL LIBRARY
Citation: Shibata Y (2017) Agenesis of the Bilateral Internal Carotid Arteries Led to the Development of Vertigo. Int J Crit Care Emerg Med 3:028. doi.org/10.23937/2474-3674/1510028 Received: July 28, 2017: Accepted: October 24, 2017: Published: October 26, 2017

Copyright: (C) 2017 Shibata Y. This is an open-access article distributed under the terms of the Creative Commons Attribution License, which permits unrestricted use, distribution, and reproduction in any medium, provided the original author and source are credited. 


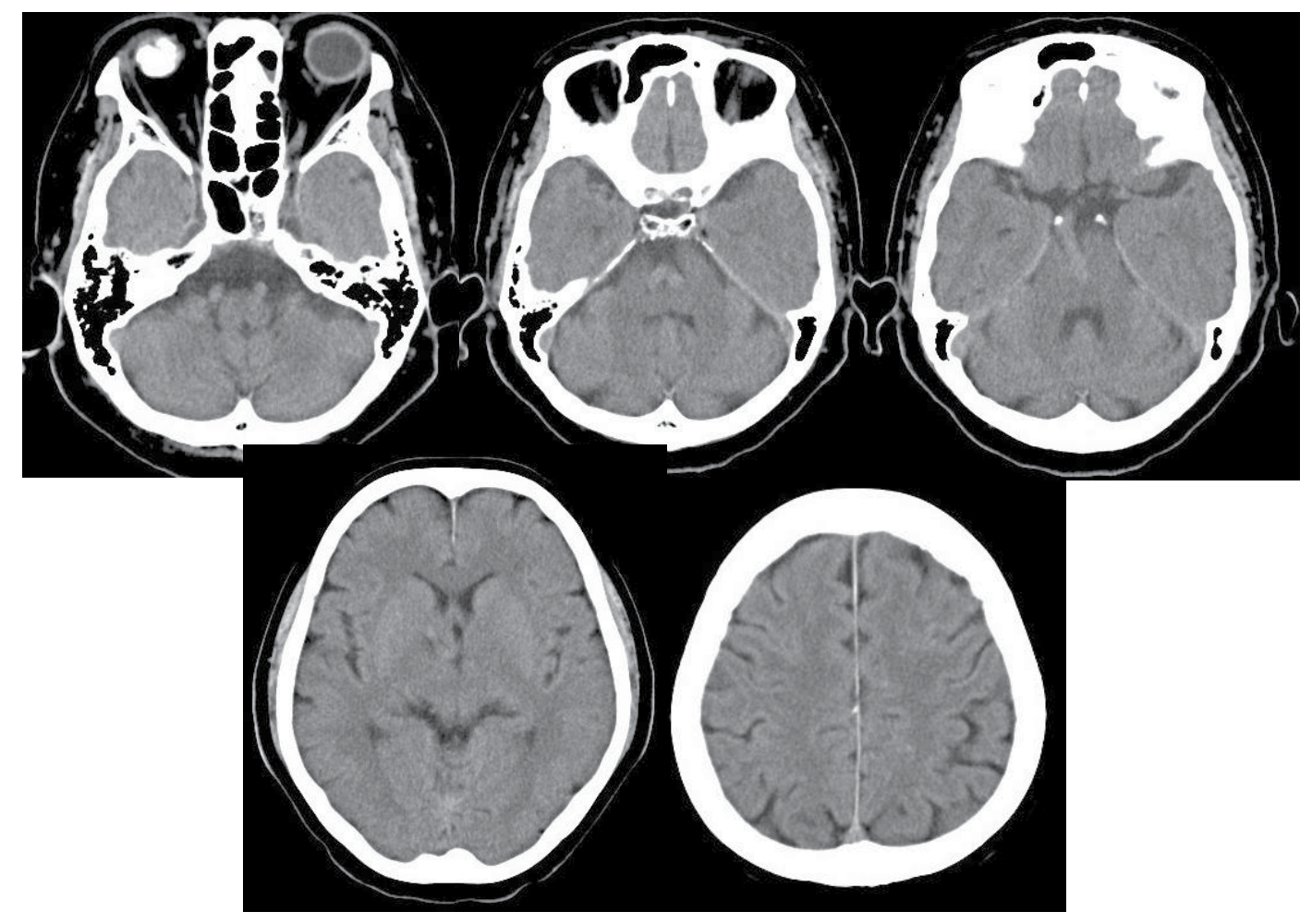

Figure 1: Brain computed tomography.

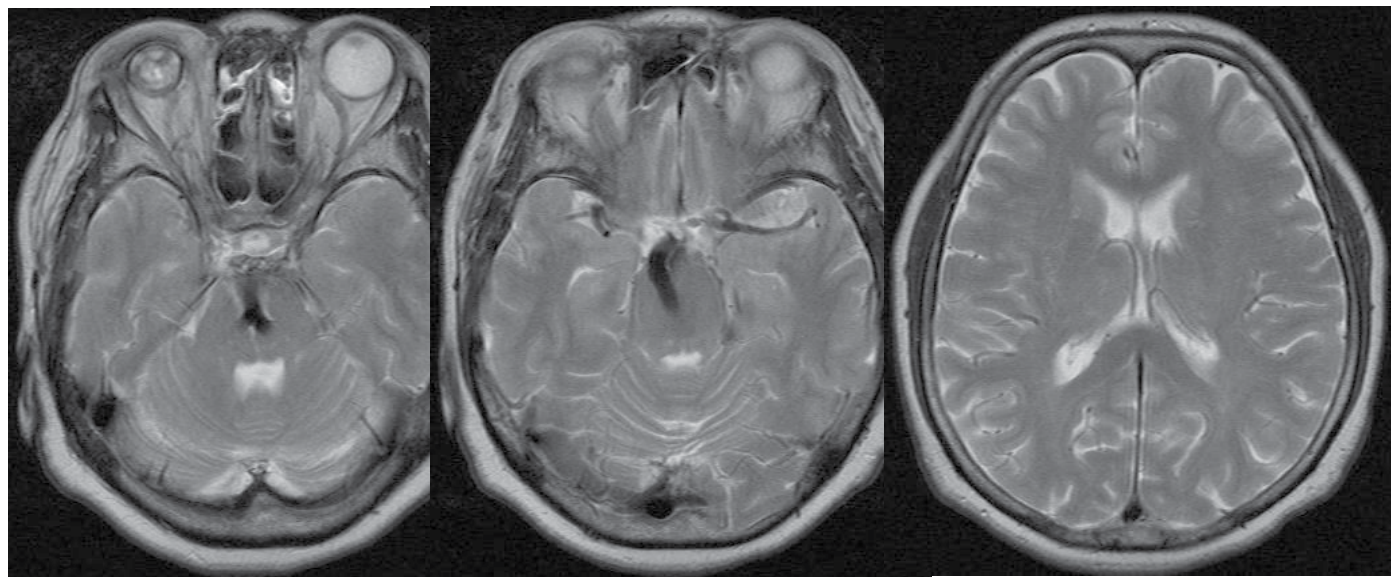

Figure 2: Magnetic Resonance (MR) imaging, T2 weighted images.

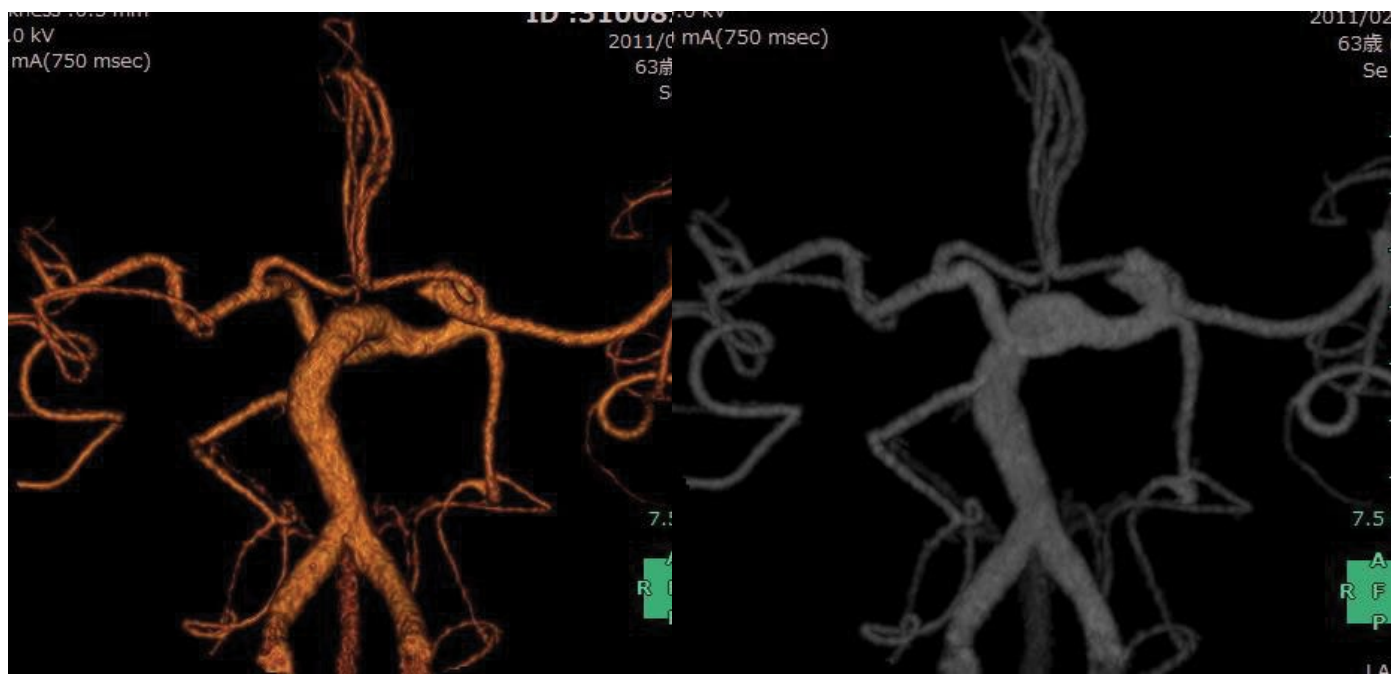

Figure 3: 3D CT angiography (Lt: volume rendering, Rt: MIP image). 


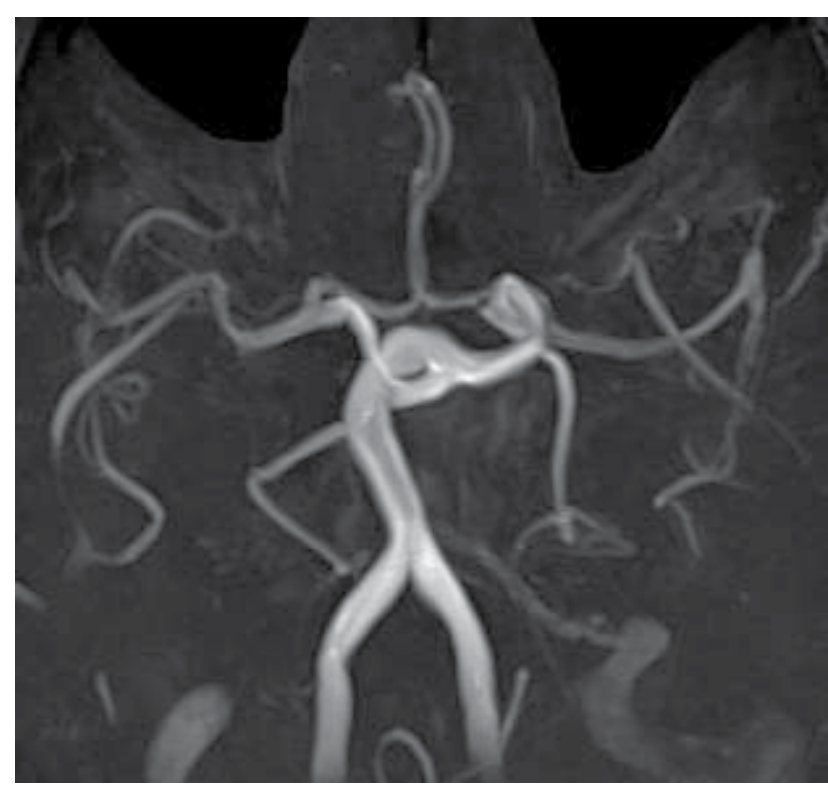

Figure 4: MR angiography, MIP image. se cases presented as subarachnoid hemorrhage caused by the rupture of a cerebral aneurysm rupture in the posterior circulation [3]. One young boy presented with two episodes of severe headache [1]. Although no intracranial aneurysm was observed, subarachnoid hemorrhage was suggested. In one patient, bilateral internal carotid artery agenesis manifested as intracerebellar hemorrhage [4].

There is one reported case of a patient who presented with dizziness due to cerebellar infarction [2]. Some patients demonstrated incidental headache without cerebrovascular accident [5].

Some cases were diagnosed as occlusion of the bilateral internal carotid arteries [6]. Agenesis and occlusion should be differentiated by bone window CT. In cases involving congenital agenesis of the bilateral internal carotid arteries, both of the carotid canals in the skull base are absent and occluded. For patients with acqui-
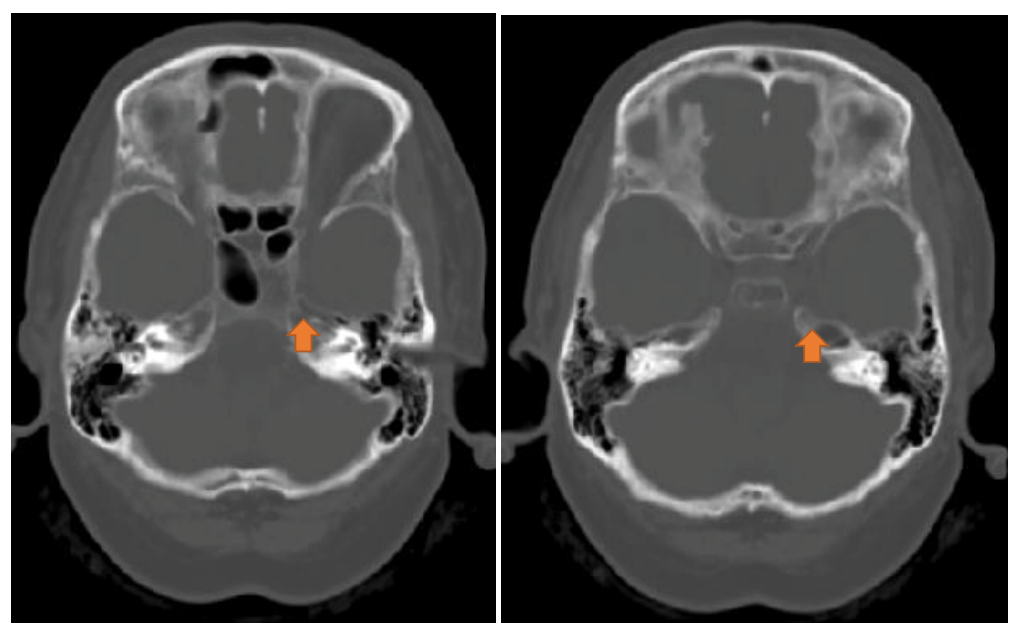

Figure 5: Skull CT (absence of carotid canal at arrows).
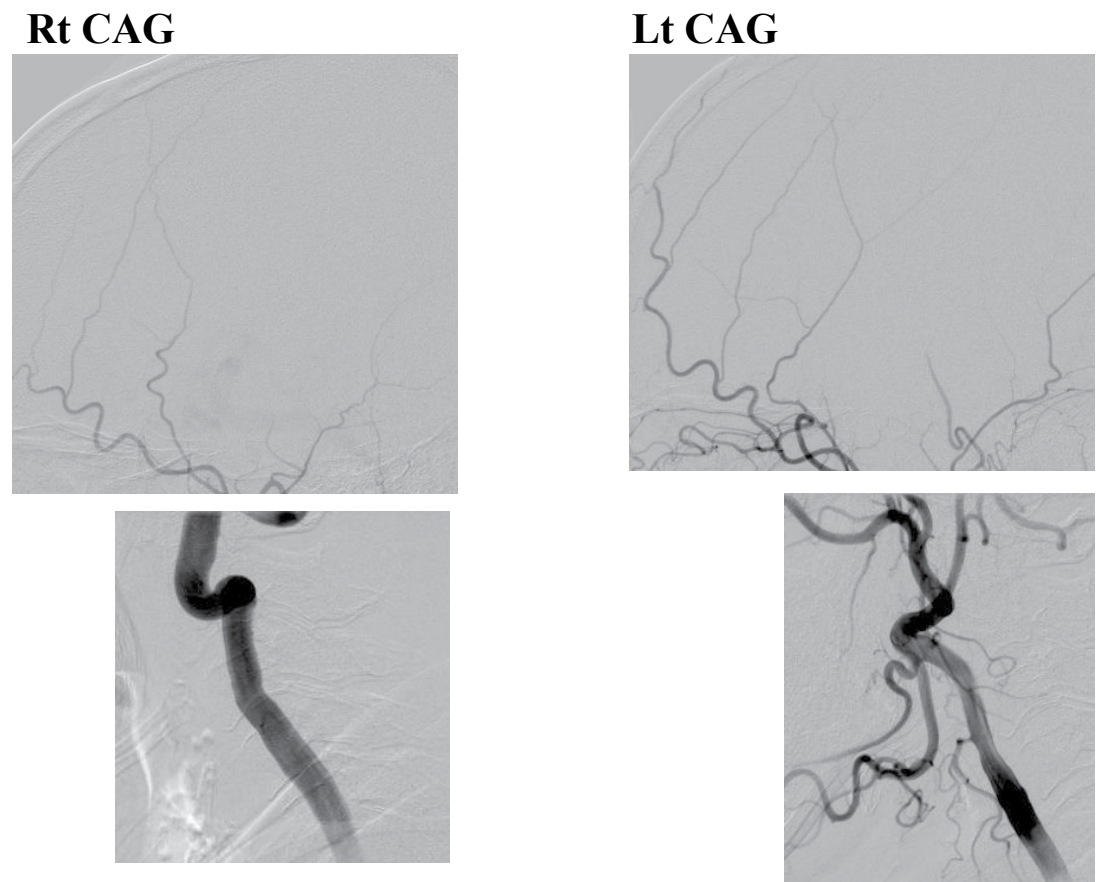

Figure 6: Cerebral angiography-1 (Lt: Rt carotid angiography, Rt: Lt carotid angiography, lateral view). 


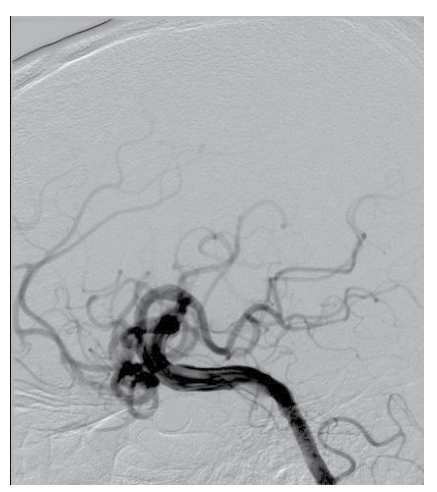

Lt VAG Lat
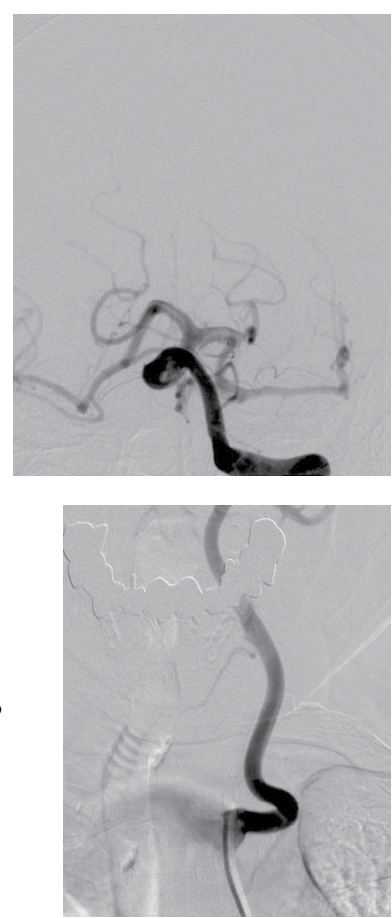

Figure 7: Cerebral angiography-2 (Lt vertebral angiography, Lt: lateral view, Rt: AP view).
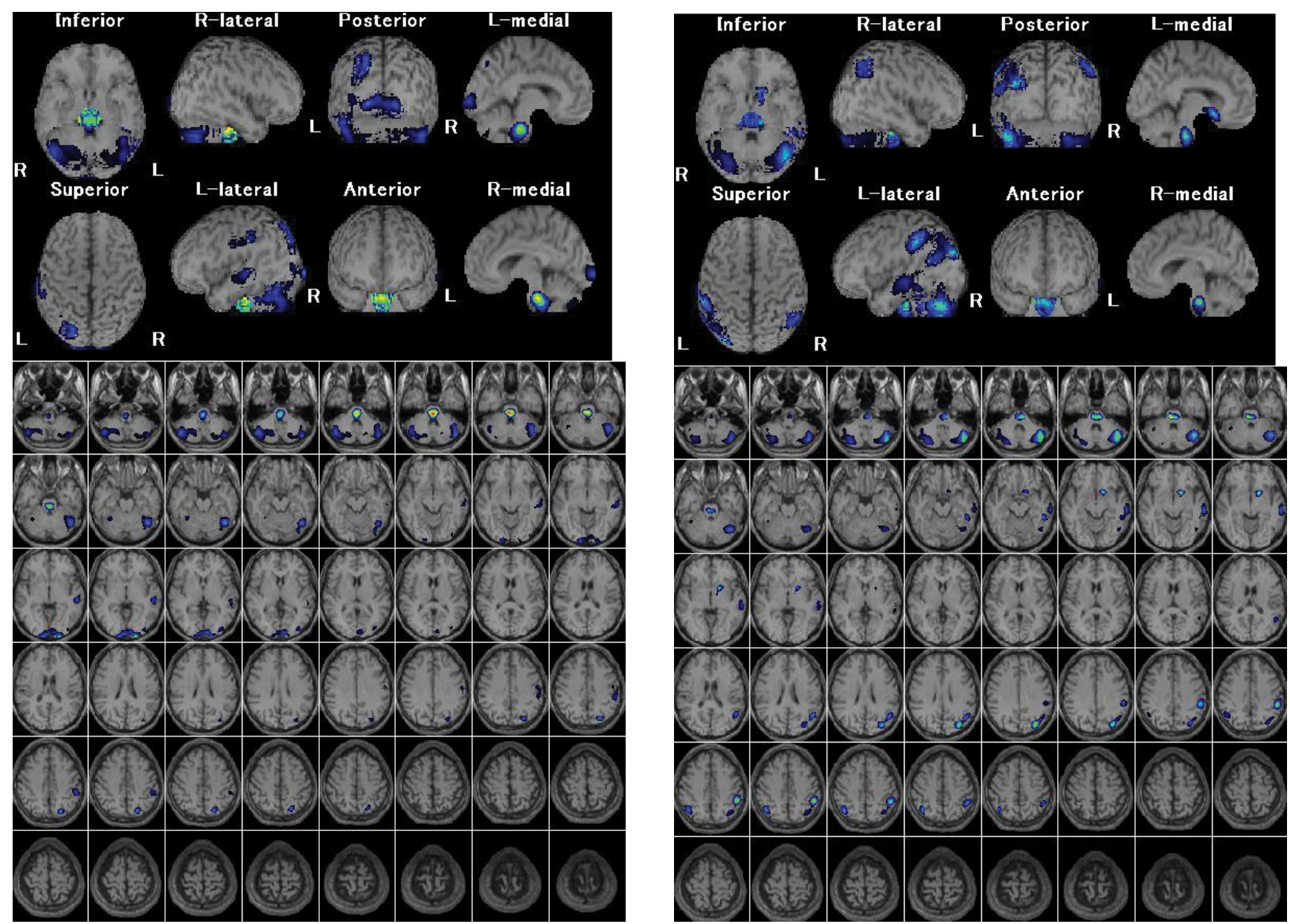

Figure 8: Tc ECD single photon emission CT (Lt: at rest, Rt: Diamox image).

red occlusion of the bilateral internal carotid arteries, both the carotid canals in the skull base are present and patent. Most reported cases of agenesis of the bilateral internal carotid arteries demonstrated occluded bilateral carotid canals in the skull base on skull CT [1,3-5].
Our case was very rare because the patient was diagnosed with agenesis of the bilateral internal carotid arteries before the development of major cerebrovascular disease. Only one patient has demonstrated occasional vertigo in association with bilateral carotid artery 


\section{CBV}

CBF

MTT

TTP

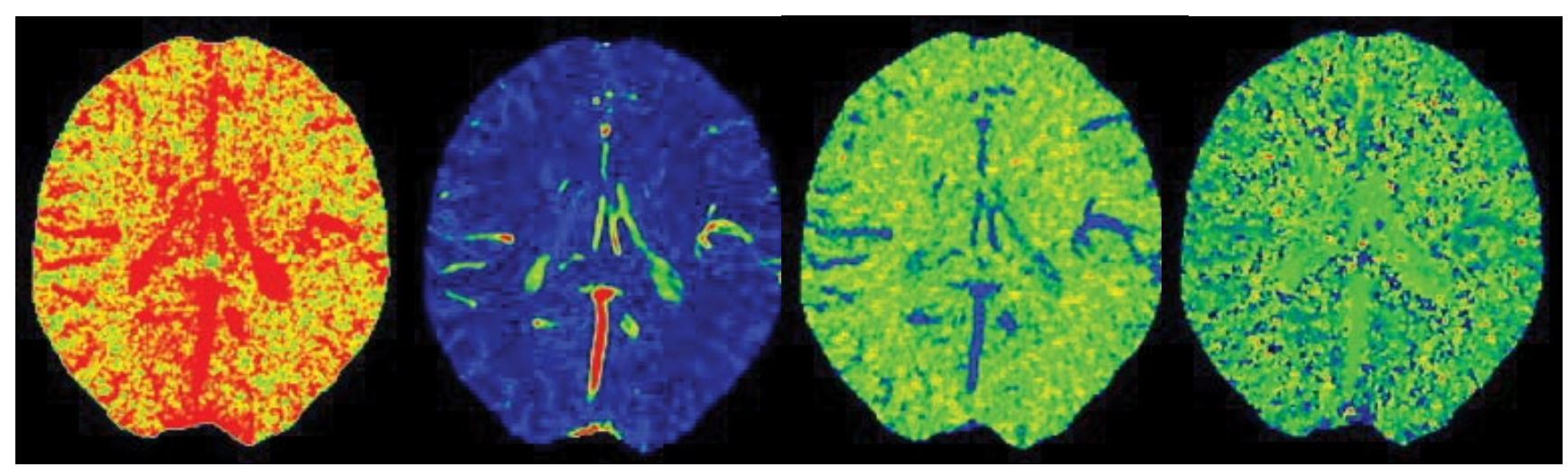

Figure 9: CT perfusion, Cerebral Blood Volume (CBV), Cerebral Blood Flow (CBF), Mean Transient Time (MTT), Time to Peak (TTP) images.

agenesis without cerebrovascular accident; however, the patient had mental retardation and corpus callosum hypogenesis [6].

There are some possible mechanisms of vertigo in our case: one is direct pulsatile pontine compression by the elongated basilar artery; another is transient or chronic cerebral ischemia caused by abnormal circulation. No cerebral infarction was revealed; however, SPECT demonstrated low perfusion at the left parietal cortex. Appropriate management of patients with agenesis of the bilateral internal carotid arteries should be discussed after the study of the natural course and a randomized therapeutic interventional trial. However, because these pathological conditions are very rare, a large study is difficult. Thus, this kind of case report may be valuable for clinical decision-making. We will closely follow up the patient and discuss therapeutic intervention to prevent the future development of major cerebrovascular disease.

\section{References}

1. Steer AC, Rowe PW (2008) Bilateral agenesis of the internal carotid artery: Case report and approach to management. J Paediatr Child Health 44: 511-513.

2. Luk YS, Man EM, Sy AN (2010) Bilateral hypoplasia of the internal carotid arteries. Singapore Med J 51: e163-e165.

3. Cruz e Silva V, Brito M (2017) Bilateral absence of the internal carotid artery and basilar aneurysm. Br J Neurosurg 31: 487-488.

4. Sunada I, Inoue T (1996) Bilateral internal carotid artery agenesis. J Neurol Neurosurg Psychiatry 61: 206-207.

5. Alexandre AM, Visconti E, Schiarelli C, Frassanito P, Pedicelli A (2016) Bilateral Internal Carotid Artery Segmental Agenesis: Embryology, Common Collateral Pathways, Clinical Presentation, and Clinical Importance of a Rare Condition. World Neurosurgery 95: 620.e9-620.e15.

6. Owada Y, Sakuta Y (1995) [Bilateral carotid artery agenesis with corpus callosum hypogenesis--a case report]. No To Shinkei 47: 589-594. 